\title{
Functionalized and Degradable Polyphthalaldehyde Derivatives
}

\author{
J. Patrick Lutz ${ }^{\dagger}$, Oleg Davydovich ${ }^{\S}$, Matthew D. Hannigan ${ }^{\dagger}$, Jeffrey S. Moore ${ }^{\S}$, Paul M. Zimmerman $^{\dagger}$, and \\ Anne J. McNeil ${ }^{+*}$
}

${ }^{\dagger}$ Department of Chemistry and Macromolecular Science and Engineering Program, University of Michigan, Ann Arbor, Michigan 48109-1055, United States

${ }^{\S}$ Department of Chemistry and Beckman Institute for Advanced Science and Technology, University of Illinois at Urbana-Champaign, Urbana, Illinois 61801, United States

\begin{abstract}
Polymers that depolymerize back to monomers can be repeatedly chemically recycled, thereby reducing their environmental impact. Polyphthalaldehyde is a metastable polymer that is rapidly and quantitatively depolymerized due to its low ceiling temperature. However, the effect of substitution on the physical and chemical properties of polyphthalaldehyde derivatives has not been systematically studied. In this work, we investigate the cationic polymerization of seven distinct o-phthalaldehyde derivatives and demonstrate that judicious choice of substituents results in materials with a wide range of ceiling temperatures (from $<-60$ to $106^{\circ} \mathrm{C}$ ) and decomposition temperatures $\left(109-196^{\circ} \mathrm{C}\right.$ ). We anticipate that these new polymers and their derivatives will enable researchers to access degradable materials with tunable thermal, physical, and chemical properties.
\end{abstract}

While much progress has been made in synthesizing polymers with diverse structures, considerably less attention has been paid to their fates after use. Depolymerizable polymers are a class of metastable materials that are readily triggered to revert back to monomers due to their low ceiling temperatures $\left(T_{\mathrm{c}}\right.$, Scheme 1$) .{ }^{1}$ Such materials have the potential to address a grand challenge in sustainability by facilitating recycling through repeated depolymerization/repolymerization cycles, extending their useful lifetimes. ${ }^{2,3}$ Depolymerizable polymers also have important applications in areas such as lithography, ${ }^{4}$ triggered release, ${ }^{5}$ and transient electronics. ${ }^{6}$

Polyphthalaldehyde (PPA) is among the most thoroughly studied depolymerizable polymers.,8 Linear or cyclic PPA (CPPA) can be obtained via anionic or cationic polymerization (respectively) of $o$-phthalaldehyde (oPA) below its $T_{c}$ of $-36^{\circ} \mathrm{C}^{9,10}$ While end-capped linear PPA and CPPA are kinetically stable at room temperature, exposure to acid results in complete depolymerization at rates too rapid to measure using standard analytical techniques (Scheme 1). ${ }^{1}$ The thermodynamic instability of PPA is key to its depolymerization, but this same property has led to challenges in polymer processing. ${ }^{11}$ For example, PPA requires plasticizers to improve its processability because its glass transition temperature $\left(T_{\mathrm{g}}\right)$ is above its thermal degradation temperature. ${ }^{12}$

A small number of substituted polyphthalaldehydes have been reported, primarily for the purpose of increasing thermal stabilities. For example, researchers at IBM found that polyphthalaldehydes bearing 4-bromo, 4-chloro, and 4-trimethylsilyl substitution exhibited higher thermal degradation temperatures than unsubstituted PPA, making them more suitable for photolithographic applications. ${ }^{13}$ In related work, Phillips and co-workers showed that endcapped linear poly(4,5-dichlorophthalaldehyde) displayed a similar effect. ${ }^{14}$ Poly(4-methylphthalaldehyde) has also been synthesized as a mechanistic probe, ${ }^{15}$ but no other PPA derivatives have been reported.

\section{Scheme 1. Low Ceiling Temperature $\left(T_{c}\right)$ Polymers}

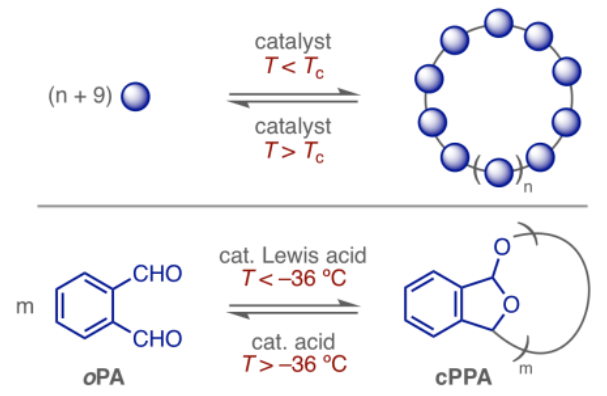

This relative lack of PPA derivatives results in part from the perception that synthesizing substituted $o$-phthalaldehydes is prohibitively challenging, relying on a few established, but often low-yielding, synthetic routes. ${ }^{7}$ Furthermore, it is still unclear a priori whether a specific phthalaldehyde is polymerizable under experimentally accessible conditions due to a lack of quantitative data on the ceiling temperatures of known PPA derivatives. To address these deficiencies, we set out to synthesize a range of substituted oPA derivatives and evaluate their Lewis acid-catalyzed polymerizations. As a result of these studies, we identified four new PPA derivatives with ceiling temperatures ranging from -23 to $+106{ }^{\circ} \mathrm{C}$ and demonstrate that their thermal degradation temperatures positively correlate with their $T_{\mathrm{c}}$. We anticipate that these new polymers will significantly expand the 
versatility of the PPA scaffold and that the resulting structure-property relationships will serve as a roadmap for researchers to develop other PPA-based materials with varied physical and chemical properties.

Computational $\boldsymbol{T}_{\mathrm{c}}$ Estimation. To estimate the ceiling temperatures for substituted phthalaldehyde derivatives, a method developed by one of our groups ${ }^{16}$ was adapted. Specifically, the acetalization of $o \mathrm{PA}$ with $\mathrm{MeOH}$ was used as the model reaction (Scheme 2, $R=H$ ). Density functional theory (B3LYP/6-31++G**) ${ }^{17}$ was used to calculate the heat of formation for each reaction component, enabling us to determine the overall enthalpy $(\Delta H)$ for the equilibrium (SI, pgs S100-S122). Using our measured $T_{\mathrm{c}}$ for oPA of $-36^{\circ} \mathrm{C}$ (vida infra), we computed the entropy $(\Delta S)$ from Eq. 1:

$$
T_{c}=\frac{\Delta H}{\Delta S}
$$

We next assumed that the $\Delta S$ determined for oPA would be similar for the substituted derivatives, given that the changes in bonding are similar for each reaction. With this assumption, the ceiling temperature was estimated by calculating the $\Delta H$ for each oPA derivative and solving Eq. 1 for $T_{\mathrm{c}}$. We chose to focus exclusively on symmetrically substituted derivatives because nonsymmetrically substituted oPA would have electronically distinct acetal linkages, making it difficult to disentangle the steric and electronic effects of different substituents.

\section{Scheme 2. Model Reaction for Phthalaldehyde Polymerization}

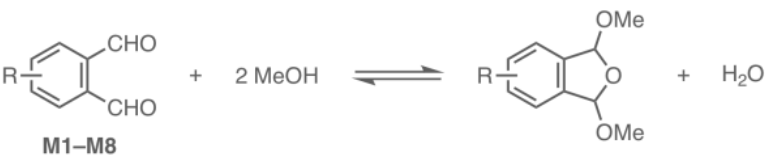

Our computations suggested that simple changes to oPA yield monomers with ceiling temperatures ranging from $122{ }^{\circ} \mathrm{C}$ to $+98{ }^{\circ} \mathrm{C}$ (Table 1). As expected, electron-donating substituents resulted in a predicted $T_{\mathrm{c}}$ below that of $\mathrm{OPA}$ (M1-M3), while electron-withdrawing substituents led to higher $T_{\mathrm{c}}$ values (M6-M8), in line with these substituents' relative abilities to impact aldehyde electrophilicity. ${ }^{14,16}$

Monomer Synthesis and Homopolymerization. We devised and executed 2-5 step synthetic routes to substituted oPAs M1-M4 and M6-M8 (SI pgs S4-S24). Notably, during purification, monomers M6-M8 underwent carbonyl hydrate formation and oligomerization to varying extents on silica, suggesting that their ceiling temperatures were near or above room temperature. In each of these cases, the dialdehyde is obtained via vacuum sublimation. ${ }^{18}$

We next subjected each monomer to $\mathrm{SnCl}_{4}$-catalyzed cationic polymerization conditions at $-78{ }^{\circ} \mathrm{C}$ in DCM. The reactions were quenched by adding pyridine to sequester the Lewis acid, then the polymers were precipitated into $\mathrm{MeOH}$ and isolated by vacuum filtration. ${ }^{9}$ As a baseline, the reaction of unsubstituted oPA (M5) produced CPPA (P5) in $35 \%$ isolated yield (Table 1, entry 5). Propoxy-substituted M1, butylthio-substituted M2, and hexyl-substituted $\mathbf{M} 3$ all possess estimated ceiling temperatures $<-78{ }^{\circ} \mathrm{C}$ and were therefore not expected to generate polymer under these reaction conditions. Consistent with the predictions, these monomers failed to generate any isolable polymer; ${ }^{1} \mathrm{H}$ NMR spectroscopic analysis following attempted precipitation from $\mathrm{MeOH}$ and solvent removal revealed mixtures of monomer and the corresponding dimethyl acetals (Table 1, entries 1-3; see also Figure S25). In contrast to M1-M3, the estimated $T_{\mathrm{c}}$ of hexynyl-substituted $\mathbf{M} 4\left(-60^{\circ} \mathrm{C}\right)$ suggested that its polymerization was feasible. Indeed, P4 was isolated in 64\% yield (entry 4). Methyl ester-substituted M6, phthalimide derivative $\mathbf{M 7}$, and tetrafluorophthalaldehyde M8 had predicted ceiling temperatures substantially higher than that of $o \mathrm{PA}$, and all three were effectively polymerized at $-78^{\circ} \mathrm{C}$ (Table 1 , entries 6-8).

Table 1. Cationic Polymerization of oPA Derivatives ${ }^{a}$

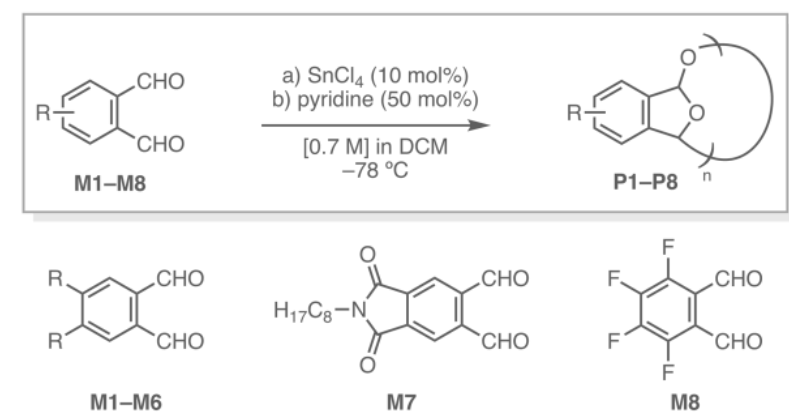

$\begin{array}{cccccc}\text { monomer } & \mathrm{R}= & \begin{array}{c}\text { est. } T_{\mathrm{c}} \\ \left({ }^{\circ} \mathrm{C}\right)\end{array} & \begin{array}{c}\text { yield }^{b} \\ (\%)\end{array} & \begin{array}{c}M_{\mathrm{n}} \\ (\mathrm{kg} / \mathrm{mol})\end{array} & \bigoplus \\ \text { M1 } & \mathrm{OPr} & -122 & 0 & - & - \\ \text { M2 } & \mathrm{SBu} & -86 & 0 & - & - \\ \text { M3 } & n-\mathrm{C}_{6} \mathrm{H}_{13} & -80 & 0 & - & - \\ \text { M4 } & \mathrm{C} \equiv \mathrm{CBu} & -60 & 64 & 12.3 & 2.2 \\ \text { M5 } & \mathrm{H} & -36^{c} & 35 & 3.2 & 2.2 \\ \text { M6 } & \mathrm{CO}_{2} \mathrm{Me} & -2 & 48 & 11.7 & 1.7 \\ \text { M7 } & - & +43 & 83 & 25.1 & 1.9 \\ \text { M8 } & - & +98 & 53 & 16.2 & 2.0\end{array}$

${ }^{a}$ Entries 4-8 represent the average of two runs. ${ }^{b}$ Isolated yield. ' Experimentally determined.

Ceiling Temperature Measurement and Thermal Analysis. While the results of the polymerizations were qualitatively consistent with our $T_{\mathrm{c}}$ estimations, we sought to evaluate these predictions quantitatively. To do so, a variable-temperature ${ }^{1} \mathrm{H}$ NMR spectroscopic method was adapted from a protocol by Kohl and co-workers (SI pgs 7297). ${ }^{19}$ Solutions with known initial concentrations of monomer $\left([\mathrm{M}]_{0}\right)$ and catalyst were prepared. Then, the monomer concentration ([M]) was measured at different temperatures by integrating the aldehyde $\mathrm{C}-\mathrm{H}$ resonance versus an internal standard. Consistent with the predicted ceiling temperatures, we observed dramatic differences in the monomer conversion based on the phthalaldehyde substitution: hexyl-substituted M3 showed $<5 \%$ conversion at $-60{ }^{\circ} \mathrm{C}$, whereas tetrafluorophthalaldehyde $\mathbf{M} 8$ reached $>95 \%$ conversion at rt (Figure 1). In the absence of a Lewis basic quenching agent, the polymerizations were reversible; warming the reactions to the starting temperatures regenerated the dialdehyde monomers. 


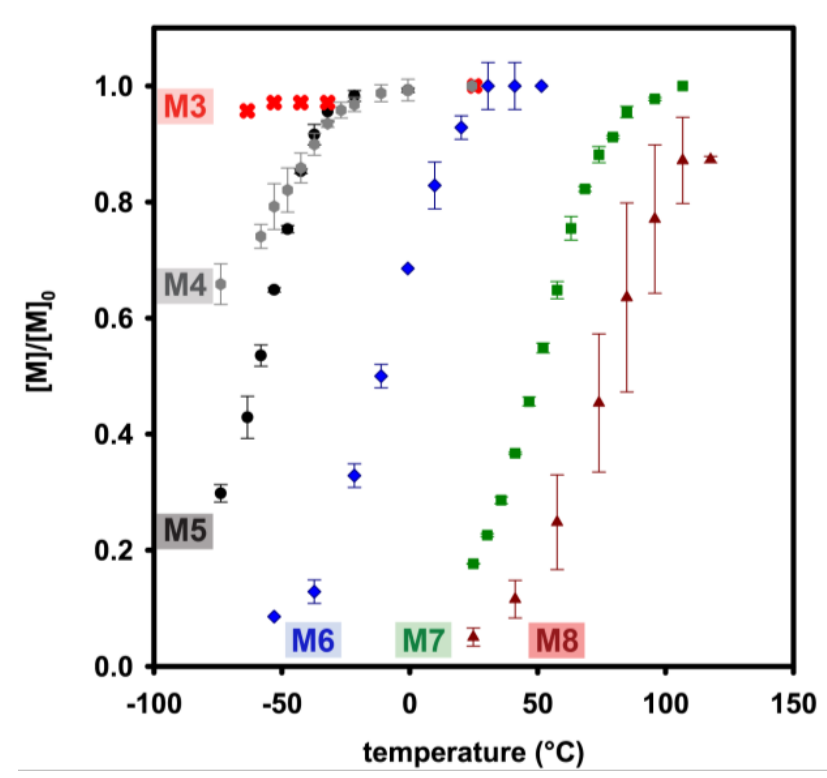

Figure 1. Plot of the normalized monomer concentrations versus temperature in the presence of $10 \mathrm{~mol}_{0} \mathrm{SnCl}_{4}$. ([M6 $]_{0}=0.35 \mathrm{M}$; $[\mathrm{M}]_{0}=0.70 \mathrm{M}$ for all other monomers.)

To quantitatively determine the ceiling temperature, the data from each reaction were plotted as $R \bullet \ln [\mathrm{M}]$ versus $1 / T$, where $R$ is the universal gas constant. The slope and intercept of the resulting line correspond respectively to the $\Delta H$ and $\Delta S$ for the polymerization. From these two values, Eq. 2 was used to calculate the experimental $T_{\mathrm{c}}$ (Table 2, entries 4-8). ${ }^{20}$ Performing this analysis on oPA provided a ceiling temperature of $-36{ }^{\circ} \mathrm{C}$ (Table 2 , entry 5 ), in close agreement with the results of Kohl and co-workers. ${ }^{19}$ We could not measure $T_{\mathrm{c}}$ values for $\mathbf{M} \mathbf{1}-\mathbf{M} \mathbf{3}$ due to the limited temperature range of the NMR probe; however, $-60{ }^{\circ} \mathrm{C}$ provides an upper limit (Table 2, entries 1-3).

$$
T_{c}=\frac{\Delta H}{\Delta S+R \cdot \ln [\mathrm{M}]_{0}}
$$

Contrary to our computational predictions, hexynylsubstituted $\mathbf{M} 4$ displayed a $T_{\mathrm{c}}$ of $-23^{\circ} \mathrm{C}$, slightly above that of oPA. This result is consistent with the weak electronwithdrawing nature of alkynyl groups ${ }^{21}\left(\sigma_{\mathrm{m}}=0.21, \sigma_{\mathrm{p}}=0.03\right.$ for $-\mathrm{C} \equiv \mathrm{CMe}) .^{22}$ Ester-substituted $\mathbf{M} 6$ exhibited a $T_{\mathrm{c}}$ of $13^{\circ} \mathrm{C}$, consistent with the stronger electron-withdrawing abilities of esters $\left(\sigma_{m}=0.36, \sigma_{p}=0.45\right.$ for $\left.-\mathrm{CO}_{2} \mathrm{Me}\right) .^{23}$ Both phthalimide $\mathbf{M 7}$ and tetrafluorophthalaldehyde $\mathbf{M} 8$ had ceiling temperatures significantly higher than room temperature $\left(74\right.$ and $106^{\circ} \mathrm{C}$, respectively). These results are attributed to the even stronger resonance- and inductivewithdrawing effects of these substituents, which increase aldehyde electrophilicity.

While our estimations of ceiling temperatures were qualitatively accurate and useful in identifying a range of substrates to examine, there are substantial quantitative differences between computed and experimental values. Examining the thermodynamic parameters in Table 2 reveals that a contributing factor in this discrepancy was our flawed assumption that the $\Delta S$ would remain approximately constant for the different monomers. In fact, a $\sim 7$-fold difference in $\Delta S$ was observed for M4 versus M8. The rationale for this large entropy difference remains unclear at this time.

Table 2. Thermodynamic Parameters for Polymerizing M1-M8 ${ }^{a}$

\begin{tabular}{|c|c|c|c|c|}
\hline $\begin{array}{c}\text { monome } \\
r\end{array}$ & $\begin{array}{l}\text { est. } T_{\mathrm{c}} \\
\left({ }^{\circ} \mathrm{C}\right)\end{array}$ & $\begin{array}{c}\Delta H \\
(\mathrm{kcal} / \mathrm{mol})\end{array}$ & $\begin{array}{c}\Delta S \\
(\mathrm{cal} / \mathrm{mol} \bullet \mathrm{K})\end{array}$ & $\begin{array}{l}\text { expt. } T_{\mathrm{C}} \\
\qquad\left({ }^{\circ} \mathrm{C}\right)\end{array}$ \\
\hline$M 1^{b}$ & -122 & - & - & $<-60$ \\
\hline $\mathrm{M}^{b}{ }^{b}$ & -86 & - & - & $<-60$ \\
\hline$M 3^{b}$ & -80 & - & - & $<-60$ \\
\hline$M 4^{b}$ & -60 & -0.90 & -2.9 & -23 \\
\hline $\mathrm{M}^{b}$ & $\mathrm{n} / \mathrm{a}$ & -3.0 & -11.9 & -36 \\
\hline$M 6^{c}$ & -2 & -4.9 & -14.9 & +13 \\
\hline $\mathrm{M}^{d}$ & +43 & -7.0 & -19.3 & +74 \\
\hline$M 8^{d}$ & +98 & -7.9 & -20.1 & +106 \\
\hline
\end{tabular}

${ }^{a}$ Entries 4-8 represent the average of two runs. ${ }^{b}$ Reaction run in $\mathrm{CD}_{2} \mathrm{Cl}_{2}$. ${ }^{c}$ Reaction run in $\mathrm{CDCl}_{3}$. ${ }^{d}$ Reaction run in 1,1,2,2-tetrachloroethane- $d_{2}$.

Following purification, ${ }^{11}$ the polymers' thermal decomposition temperatures $\left(T_{\mathrm{d}}\right)$ were measured by differential scanning calorimetry (DSC; SI pgs S49-S71). ${ }^{24} \mathrm{~A}$ positive relationship between $T_{\mathrm{c}}$ and $T_{\mathrm{d}}$ was apparent, with $T_{\mathrm{d}}$ ranging from $109^{\circ} \mathrm{C}$ for $\mathrm{M} 5$ to $196{ }^{\circ} \mathrm{C}$ for M8 (Figure $2 \mathrm{~A}$ ). Interestingly, polymer $\mathbf{P 8}$ was the only polymer to exhibit a glass transition below its decomposition temperature (Figure $2 \mathrm{~B})$, suggesting that $\mathbf{P 8}$ could have processing advantages over unsubstituted PPA.
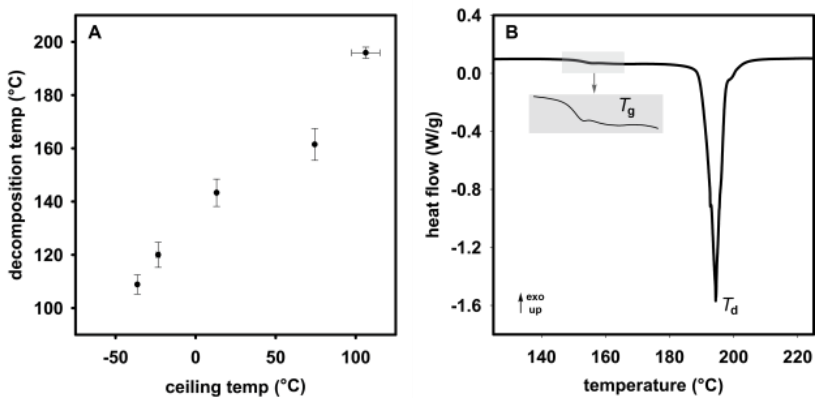

Figure 2. (A) Plot of decomposition temperature (via DSC) versus the experimental ceiling temperature for $\mathbf{P 4 - P 8}$ (via ${ }^{1} \mathrm{H}$ NMR spectroscopy). (B) DSC thermogram for $\mathbf{P 8}$.

Cationic Copolymerization. To elucidate the impact of copolymer composition on thermal stabilities, we copolymerized M5 with M6. Varying feed ratios ranging from 0-100 mol\% M6 were used (SI pgs S54-S62). ${ }^{25}$ Following purification, the cumulative copolymer composition was estimated via ${ }^{1} \mathrm{H}$ NMR spectroscopy. A linear trend between copolymer composition and feed ratio was evident (Figure $3 \mathrm{~A})$, suggesting either an alternating or statistical sequence. Thermal analysis of the copolymers revealed a near-linear relationship between copolymer composition and $T_{\mathrm{d}}$ (Figure 3B). The slight non-linearity is likely a result of differing levels of residual Lewis acid remaining in the (co)polymers after purification. ${ }^{11,26} \mathrm{~A}$ similar trend in decomposition temperature has been reported for copolymers of oPA with ethyl glyoxylate. ${ }^{12}$ These results demonstrate that PPA substitution patterns and incorporation ratios can be rationally designed to obtain copolymers with targeted thermal properties, though improvements to the purification 
process will likely be required to ensure consistent results.
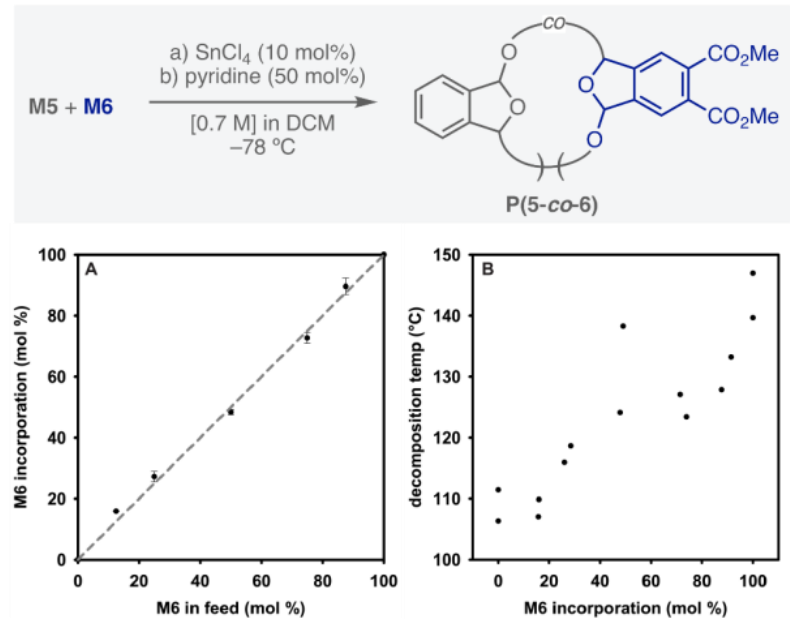

Figure 3. (A) Plot of $\mathbf{M} \mathbf{6}$ incorporation in the copolymer (via ${ }^{1} \mathrm{H}$ NMR spectroscopy) versus $\mathbf{M} 6$ composition in the feed. The dashed line is for a 1:1 relationship. (B) Plot of decomposition temperature (via DSC) versus $\mathbf{M 6}$ incorporation in copolymer.

Microcapsule fabrication. In previous work, cPPA was used to form triggerable core-shell microcapsules. ${ }^{27}$ The new PPA derivatives described herein could be used to generate capsules that exhibit different surface functionalities, specific ion coactivators, ${ }^{27 \mathrm{~b}}$ and rates of payload release. For a preliminary study, P6 was prepared on gram scale in $76 \%$ yield (SI pgs S71) and subjected to the previously described microencapsulation conditions optimized for unsubstituted CPPA (SI pgs S98-S99). ${ }^{27 a}$ Briefly, oil-in-water emulsions comprised of $\mathbf{P 6 / j o j o b a ~ o i l / D C M ~ i n ~ a q u e o u s ~ p o l y ( v i n y l ~}$ alcohol) were generated via a microfluidic flow focusing device. Rapid evaporation of the DCM followed by filtering and washing furnished microcapsules with an average diameter of $228 \pm 5 \mu \mathrm{m}$. Scanning electron microscopy (SEM) confirmed that these capsules exhibited a core-shell architecture, with an estimated shell-wall thickness of $11 \mu \mathrm{m}$ (Figure 4). These P6 microcapsules represent a potential starting point to generate anionic, cationic, and labelled depolymerizable capsules via post-capsule functionalization reactions.
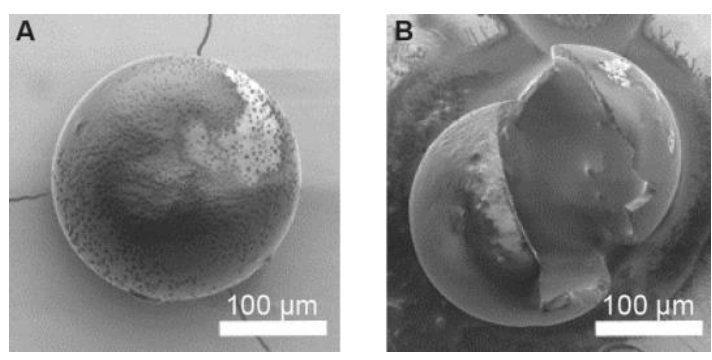

Figure 4. SEM images of microcapsules of P6.

To summarize, seven different substituted o-phthalaldehyde derivatives were synthesized and subjected to cationic polymerization conditions. As predicted computationally, several of these compounds were unreactive at $-78^{\circ} \mathrm{C}$ due to their low ceiling temperatures. At the same time, four new polyphthalaldehydes bearing alkyne, ester, imide, and fluorine substituents were successfully synthesized due to their higher ceiling temperatures. Remarkably, poly(tetrafluorophthalaldhyde) is both highly stable and likely melt-processable. We anticipate that these (co)polymers, as well as derivatives synthesized via post-polymerization modification, will enable researchers to access degradable materials with tunable thermal, physical, and chemical properties. Moreover, the combined computational and experimental method to predict and measure ceiling temperatures should help guide the synthesis of new PPA derivatives.

\section{ASSOCIATED CONTENT}

Supporting Information. Experimental details, characterization data, synthetic procedures.

\section{AUTHOR INFORMATION}

\section{Corresponding Author}

*ajmcneil@umich.edu

ORCID

Anne J. McNeil 0000-0003-4591-3308

\section{ACKNOWLEDGMENT}

We (AJM and JSM) gratefully acknowledge the National Science Foundation for supporting this work through a Phase I Center for Chemical Innovation Grant (\#1740597) as well as the Department of Defense for a National Defense Science and Engineering Graduate Fellowship for MDH.

\section{REFERENCES}

Recyclable Metastable Polymers and Composites. Chem. Mater. 2019, 31, 398-406.

${ }^{4}$ Bowden, M.J.; Thompson, L.F. Electron Irradiation of Poly(olefin sulfones). Application to Electron Beam Resists. J. Appl. Polym. Sci. 1973, 17, 3211-3221.

${ }^{5}$ Esser-Kahn, A.P.; Odom, S.A.; Sottos, N.R.; White, S.R.; Moore, J.S. Triggered Release from Polymer Capsules. Macromolecules 2011, 44, 5539-5553. 
${ }^{6}$ Fu, K.K.; Wang, Z.; Dai, J.; Carter, M.; Hu, L. Transient Electronics: Materials and Devices. Chem. Mater. 2016, 28, 3527-3539.

7 Wang, F.; Diesendruck, C.E. Polyphthalaldehyde: Synthesis, Derivatives, and Applications. Macromol. Rapid Commun. 2018, 39, 1700519.

8 (a) Aso, C.; Tagami, S. Cyclopolymerization of $o$-Phthalaldehyde. J. Polym. Sci. Part B: Polym. Lett. 1967, 5, 217-220. (b) Aso, C.; Tagami, S.; Kunitake, T. Polymerization of Aromatic Aldehydes. II. Cationic Cyclopolymerization of Phthalaldehyde. J. Polym. Sci. Part A: Polym. Chem. 1969, 7, 497-511. (c) Aso, C.; Tagami, S. Polymerization of Aromatic Aldehydes. III. The Cyclopolymerization of Phthalaldehyde and the Structure of the Polymer. Macromolecules 1969, 2, 414-419.

9 Kaitz, J.A.; Diesendruck, C.E.; Moore, J.S. End Group Characterization of Poly(phthalaldehyde): Surprising Discovery of a Reversible, Cationic Macrocyclization Mechanism. J. Am. Chem. Soc. 2013, 135, 12755-12761.

10 Ceiling temperatures ranging from -35 to $-43{ }^{\circ} \mathrm{C}$ have been reported for PPA. We use our own experimentally determined temperature of $-36^{\circ} \mathrm{C}$ throughout this manuscript.

${ }^{11}$ Feinberg, A.M.; Lopez Hernandez, H.; Plantz, C.L.; Mejia, E.B.; Sottos, N.R.; White, S.R.; Moore, J.S. Cyclic Poly(phthalaldehyde): Thermoforming a Bulk Transient Material. ACS Macro. Let. 2018, 7, 47-52.

${ }^{12}$ Kaitz, J.A.; Moore, J.S. Copolymerization of $o$-Phthalaldehyde and Ethyl Glyoxylate: Cyclic Macromolecules with Alternating Sequence and Tunable Thermal Properties. Macromolecules 2014, 47, 5509-5513.

13 (a) Ito, H.; Schwalm, R. Thermally Developable, Positive Resist Systems with High Sensitivity. J. Electrochem. Soc. 1989, 136, 241-245. (b) Ito, H.; Ueda, M.; Renaldo, A.F. Thermally Developable, Positive Tone, Oxygen RIE Barrier Resist for Bilayer Lithography. J. Electrochem. Soc. 1989, 136, 245-249.

14 (a) DiLauro, A.M.; Phillips, S. T. End-Capped Poly(4,5dichlorphthalaldehyde): A Stable Self-Immolative Poly(aldehyde) for Translating Specific Inputs into Amplified Outputs, Both in Solution and the Solid State. Polym. Chem. 2015, 6, 3252-3258. (b) DiLauro, A.M.; Lewis, G.G.; Phillips, S.T. Self-Immolative Poly(4,5-dichlorophthalaldehyde) and its Applications in Multi-Stimuli-Responsive Macroscopic Plastics. Angew. Chem. Int. Ed. 2015, 54, 6200-6205.

15 Kaitz, J.A.; Diesendruck, C.E.; Moore, J.S. Dynamic Covalent Macrocyclic Poly(phthalaldehyde)s: Scrambling Cyclic Homopolymer Mixtures Produces Multi-Block and Random Cyclic Copolymers. Macromolecules 2013, 46, 8121-8128.

${ }^{16}$ Kaitz, J.A.; Moore, J.S. Functional Phthalaldehyde Polymers by Copolymerization with Substituted Benzaldehydes. Macromolecules 2013, 46, 608-612.
17 Shao, Y. et al. Advances in Molecular Quantum Chemistry Contained in the Q-Chem 4 Program Package. Mol. Phys. 2015, 113, 184-215.

18 Monomer M8 was isolated in $~ 80 \%$ purity, presumably due to oligomerization during vacuum sublimation as a result of its high electrophilicity.

19 Schwartz, J.M.; Engler, A.; Phillips, O.; Lee, J.; Kohl, P.A. Determination of Ceiling Temperature and Thermodynamic Properties of Low Ceiling Temperature Polyaldehydes. J. Polym. Sci. Part A: Polym. Chem. 2017, 56, 221-228.

${ }^{20}$ Dainton, F.S.; Ivin, K.J. Reversibility of the Propagation Reaction in Polymerization Processes and its Manifestation in the Phenomenon of a 'Ceiling Temperature.' Nature 1948,162, 705-707.

${ }^{21}$ Bizier, N.P.; Wackerly, J.Wm.; Braunstein, E.D.; Zhang, M.; Nodder, S.T.; Carlin, S. M.; Katz, J.L. An Alternative Role for Acetylenes: Activation of Fluorobenzenes toward Nucleophilic Aromatic Substitution. J. Org. Chem. 2013, 78, 5987-5998.

${ }^{22}$ Charton, M. Electrical Effect Substituent Constants for Correlation Analysis. In Progress in Physical Organic Chemistry; Taft, R.W., Ed.; Wiley: New York, 1981; Vol. 13; pp 119-251.

23 Little, W.F.; Reilley, C.N.; Johnson, J.D.; Lynn, K.N.; Sanders, A.P. Chronopotentiometric Studies of Ferrocene Derivatives. I. Determination of Substituent Constants with Substituted Phenylferrocenes. J. Am. Chem. Soc. 1964, 86, 1376-1381.

24 Thermogravimetric analysis was unsuitable, as the $T_{\mathrm{d}}$ for some polymers was above or similar to the monomer volatilization temperature.

${ }^{25}$ Copolymers of $O P A$ with monoaldehydes have been reported. See refs. 12, 16, and (a) Schwartz, J.M.; Gourdin, G.; Phillips, O.; Engler, A.; Lee, J.; Abdulkadir, N.R.; Miller, R.C.; Sutlief, A.; Kohl, P.A. Cationic Polymerization of High-Molecular Weight Phthalaldehyde-Butanal Copolymer. J. Appl. Polym. Sci. 2019, 136, 46921. (b) Engler, A.; Phillips, O.; Miller, R.C.; Tobin, C.; Kohl, P.A. Cationic Copolymerization of $o$-Phthalaldehyde and Functional Aliphatic Aldehydes. Macromolecules 2019, 52, 4020-4029.

${ }^{26}$ Schwartz, J.M.; Phillips, O.; Engler, A.; Sutlief, A.; Lee, J.; Kohl, P.A. Stable, High-Molecular-Weight Poly(phthalaldehyde). J. Polym. Sci. Part A: Polym. Chem. 2017, 55, 1166-1172.

27 (a) Tang, S.; Yourdkhani, M.; Possanza Casey, P.M.; Sottos, N.R.; White, S.R.; Moore, J.S. Low-Ceiling-Temperature Polymer Microcapsules with Hydrophobic Payloads via Rapid EmulsionSolvent Evaporation. ACS Appl. Mater. Interfaces 2017, 9, 20115-20123. (b) Tang, S.; Tang, L.; Lu, X.; Liu, H.; Moore, J.S. Programmable Payload Release from Transient Polymer Microcapsules Triggered by a Specific Ion Coactivation Effect. J. Am. Chem. Soc. 2018, 140, 94-97. 
structure-property relationships

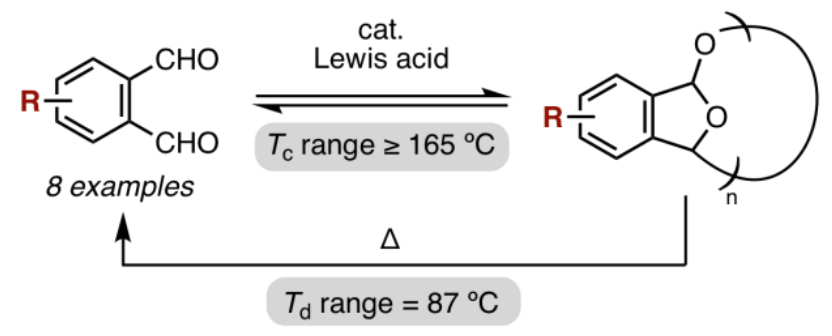

